\title{
Discrimination of bile acids by the rainbow trout olfactory system: Evidence as potential pheromone
}

\author{
PERCILIA C GIAQUINTO $^{1 *}$ and TOSHIAKI J HARA ${ }^{2,3}$
}

\author{
${ }^{1}$ Physiology Department, Biological Institute, Universidade Estadual Paulista, Botucatu, São Paulo, Brazil \\ ${ }^{2}$ Freshwater Institute, Canada Department of Fisheries and Oceans, Winnipeg, Manitoba, Canada \\ ${ }^{3}$ Department of Zoology, University of Manitoba, Winnipeg, Manitoba
}

\begin{abstract}
Electro-olfactogram recording was used to determine whether the olfactory epithelium of adult rainbow trout is specifically sensitive to bile acids, some of which have been hypothesized to function as pheromones. Of 38 bile acids that had been pre-screened for olfactory activity, 6 were selected. The rainbow trout-specific bile acids, taurocholic acid (TCA), and taurolithocholic acid 3-sulfate (TLS) were the most potent compounds tested. TLS had a distinctive dose-response curve. Cross-adaptation experiments demonstrated that sensitivity to bile acids is attributable to at least 3 independent classes of olfactory receptor sites. Our data suggest that bile acids are discriminated by olfaction in rainbow trout, supporting the possibility that these compounds function as pheromones.
\end{abstract}

Key terms: bile acids, chemical signals, electro-olfactogram, olfaction, pheromones, rainbow trout.

\section{INTRODUCTION}

Bile acids and amino acids, together with sex steroids and prostaglandins, comprise four major classes of chemicals that have been identified as specific olfactory stimulants for fishes and their stimulatory effectiveness characterized in over 30 species (Hara, 1994a; Hara, 1994b).

Extreme olfactory sensitivities to bile acids, coupled with their wide distribution and chemical variations have been implicated for their role in fish behavior (Dolving et al.,1980; Hara et al., 1984; Sola and Tosi, 1993; Zhang et al., 2001). Although the gustatory function of bile acids has yet to be ascertained, behavioral evidence suggests that one of their functions as olfactory stimulants may be to serve as pheromones for migratory anadromous fishes, some of which appear to recognize and select the odor of conspecifics when choosing spawning streams (Doving et al., 1980; Stabell,
1992). Studies using electroencephalogram recording showed that bile acids are potent olfactory stimulants for the grayling and Arctic charr (Doving et al., 1980). Taurocholic acid (TCA) is a potent olfactory stimulus to several salmonids (Hara et al, 1984; Quinn and Hara, 1986; Zhang and Hara, 1991; Hara and Zhang, 1996) and goldfish (Sorensen et al., 1987).

In teleosts, the principal biliary bile acids are (1) sulphated bile alcohol, mainly 5-cyprinol and 5-chimaerol; and (2) $\mathrm{C}_{24}$ bile acids, mainly cholic acid (CA), chenodeoxycholic acid (CD), deoxycholic acid (DC), and haemulcholic acid (Haslewood, 1967; Goto et al., 1996). The $\mathrm{C}_{24}$ bile acids are taurine amidated and/or sulphated. Also, cysteinolic acid-amidated bile acids were found in the bile of some marine species (Une et al, 1991; Goto et al, 1996). In the bile acid composition of rainbow trout, cholic acid was found to be the main component and constituted over $85 \%$ of total (Denton and Yousef, 1974).

\footnotetext{
* Corresponding Author: Percilia Giaquinto, Fazenda Surubin, Caixa Postal 11, Macatuba, São Paulo, Brazil 17290000; Email: perciliag@gmail.com.
} 
Chenodeoxycholic acid accounted for $14 \%$ and $3 \alpha \pm, 12 \alpha \pm-7-k e t o$ and $7 \alpha \pm, 12 \alpha \pm-3-$ keto- $5 \beta \leq$ cholanoates for $1 \%$ or less of total. Based on these findings, we planned to investigate a possible pheromonal role of bile acids in rainbow trout, determining which of them would be detected by olfaction.

Despite growing interest in the role of bile acids in fish chemoreception (Zhang et al., 2001), little effort has been made to examine structure-activity relationships and receptor specificity for bile acids in the fish olfactory system. Analyses of this interaction will reveal the relative effectiveness of stimulants interacting with the putative receptors and, consequently, will provide information on whether a particular stimulus will be effectively detected by fish and which structural features are required for a stimulant to interact with the receptors.

To investigate a possible pheromonal role of bile acids in rainbow trout, our studies focused on: 1) which bile acids are detected by olfaction; and 2) how bile acids are detected by olfaction, using electroolfactogram (EOG) recordings. The EOG, or transepithelial voltage transient, represents a summation of generator potential of olfactory neurons (Ottoson, 1971). EOG responses of fish are likely dependent upon activities of a large population of neurons over an entire area, if not the whole olfactory rosette, rather than the group of receptor cells on a single lamella in close proximity to the recording electrode. The high sensitivity and stability of EOG has proven advantageous for monitoring the olfactory sensitivity to odorants. The current study uses standard electrophysiological competition (cross-adaptation) procedures to determine whether bile salt odorants compete for shared odorant receptors.

\section{MATERIAL AND METHODS}

\section{Experimental animals}

Rainbow trout (Oncorhynchus mykiss) were obtained from a Manitoba provincial fish hatchery and maintained in the Freshwater
Institute, Department of Fisheries and Oceans, Winnipeg, Canada. Fishes were fed with commercial food pellets and supplied with flowing, aerated dechlorinated Winnipeg city water at $11.5-12.5^{\circ} \mathrm{C}$ under a 16L: 8D photoperiod. Experimental procedures employed in the studies complied with the guidelines issued by the Canadian Council for Animal Care.

\section{Stimulus administration and EOG recording}

Test fish were tranquilized with MS222 (1: 8000), anaesthetized intraperitoneally with amobarbital (30 $\mathrm{mg} / \mathrm{Kg}$ body weight), and immobilized with an intramuscular injection of Flaxedil (gallamine triethiodide, 3-4 mg/Kg body weight). The anaesthetized fish was wrapped with an absorbent tissue and secured in a holding apparatus. The right-side rosette of the olfactory sac was exposed by removing the dorsal aspect of the skin and the cartilage of the olfactory sac. The naris and the gills were perfused with dechlorinated plain water. To deliver plain water or stimulants to the naris, the method of Sveinsson and Hara (1990) was used. Briefly, a pneumatic activator switches plain water to/from the stimulant solution delivered by two identical polyethylene tubes. The pneumatic valve was controlled by a solenoid and an associated electronic timing device (Hara et al., 1973). The solution flowed through a glass capillary positioned over the rosette (10 $\mathrm{ml} / \mathrm{min})$. This system provides an approximate square stimulation at required concentration each time with no apparent interruption or disturbance of the flow to the naris. The stimulus duration used was $10 \mathrm{~s}$.

EOG responses were recorded with a saline-gelatin (8\%) filled capilary (Type diameter 100-150 $\mu \mathrm{m})$ bridged to an $\mathrm{AgAgCl}$ eletrode (Type MEH-15, World Precision Instruments, Sarasota, FL, USA) filled with $3 \mathrm{M} \mathrm{KCl}$ and recorded on a polygraph (Model 79, Grass Instrument, West Warwick, USA). A reference electrode of the same type was placed lightly on the dorsal skin surface adjacent to the perfused olfactory cavity. The 
recording electrode was positioned near the central ridge, posterior portion of the rosette, and slightly above the olfactory epithelium.

\section{Cross-adaptation}

Cross-adaptation experiments (Caprio and Byrd, 1984) were conducted to compare the EOG response to a test stimulus before and during adaptation to an adapting compound using a protocol by Sveinsson and Hara (1990). A standard test series measured a competitor odorant's effect on the responses to each test odorant. The 3 phases of a standard test series are: phase 1, measurement of the response to a test odorant when plain water bathes the olfactory epithelium; phase 2 , measurement of the response to a test odorant when a competitor odorant bathes the olfactory epithelium (the olfactory epithelium was bathed by a competitor odorant for approximately $60 \mathrm{~s}$ prior to first application of the test odorant); and phase 3 , the flow bathing the olfactory epithelium is returned to plain water, and the odorant is re-tested to confirm that adaptation is reversible.

\section{Chemicals}

Bile acids and amino acids were purchased from Steraloids (Newport, RI, USA) or Sigma Chemical (St. Louis, MO, USA). Trivial names for bile acids are used throughout the text. Names and abbreviations of the most potent bile acids are listed in Table 1 ( 6 out 32 tested).

Stock solutions of the stimulants $\left(10^{-3} \mathrm{M}\right.$ concentrations) were prepared with distilled water and stored in a refrigerator. Test solutions were formulated immediately before testing by diluting with plain water. To eliminate the effect of distilled water, aliquots of stock solutions were diluted at least 100 times with plain water to form test formulas.

\section{Data analysis}

A one-way ANOVA followed by a Dunnett post-hoc test upon significance $(P-0.05)$ was performed on the percentage of unadapted responses obtained in the crossadaptation experiments. A significant response in the Dunnett test between the stimulation with the chemical used as adaptant and another stimulant was taken as evidence of the activation of distinct receptor sites.

All EOG responses were normalized as relative magnitudes of the standard reference stimulus $10^{-5} \mathrm{M}$ L-Serine. All data are presented as means \pm SEM. Differences between groups of means were determined using one-way analyses of variance. The t-test or a paired t-test, when appropriate, was used to determine differences between means from two groups. A $p$ value $<0.05$ was used to identify significant differences between groups.

TABLE I

Names and abbreviations for the bile acids tested

\begin{tabular}{|c|c|c|}
\hline Abbreviation & Trivial name & Chemical name \\
\hline $\mathbf{C A}$ & Cholic acid & $3 \alpha \pm, 7 \alpha \pm, 12 \alpha \pm$-Trihydroxy-5 $\beta \leq$-cholan-24-oic acid \\
\hline CD & Chenodeoxycholic acid & $3 \alpha \pm, 7 \alpha \pm$-Dihydroxy-5 $\beta \leq-$ cholan-24-oic acid \\
\hline DC & Deoxycholic acid & $3 \alpha \pm, 12 \alpha \pm$-Dihydroxy-5 $\beta \leq$-cholan-24-oic acid \\
\hline TCA & Taurocholic acid & $\begin{array}{l}3 \alpha \pm, 7 \alpha \pm, 12 \alpha \pm \text {-Trihydroxy-5 } \beta \leq \text {-cholan-24-oic acid N- } \\
\text { (2-sulfoethy)amide }\end{array}$ \\
\hline TCD & Taurochenodeoxycholic acid & $\begin{array}{l}3 \alpha \pm, 7 \alpha \pm \text {-Dihydroxy-5 } \beta \leq \text {-cholan-24-oic acid } \\
\text { N-(2-sulfoethy)amide }\end{array}$ \\
\hline TLS & Taurolithocholic acid $3 \alpha \pm$-sulphate & $\begin{array}{l}3 \alpha \pm \text {-Hydroxy- } 5 \beta \leq \text {-cholan-24-oic acid } \\
\mathrm{N} \text {-(2-sulfoethy)amide }\end{array}$ \\
\hline
\end{tabular}




\section{RESULTS}

\section{Characteristics of EOG responses}

The EOG response to a bile acid was a rapid negative response reaching its peak amplitude 1-2 s after the stimulation switch was turned on. Then, the response declined exponentially to a maintained tonic level throughout the 10-s stimulus period. Upon cessation of the stimulus, the potential returned quickly to the baseline. As the stimulant concentration increased, the time of latency phase became gradually shorter, whereas the time of recovery phase became longer. Figure 1 shows the tonic/phasic responses ratios for the six most representative bile acids tested.
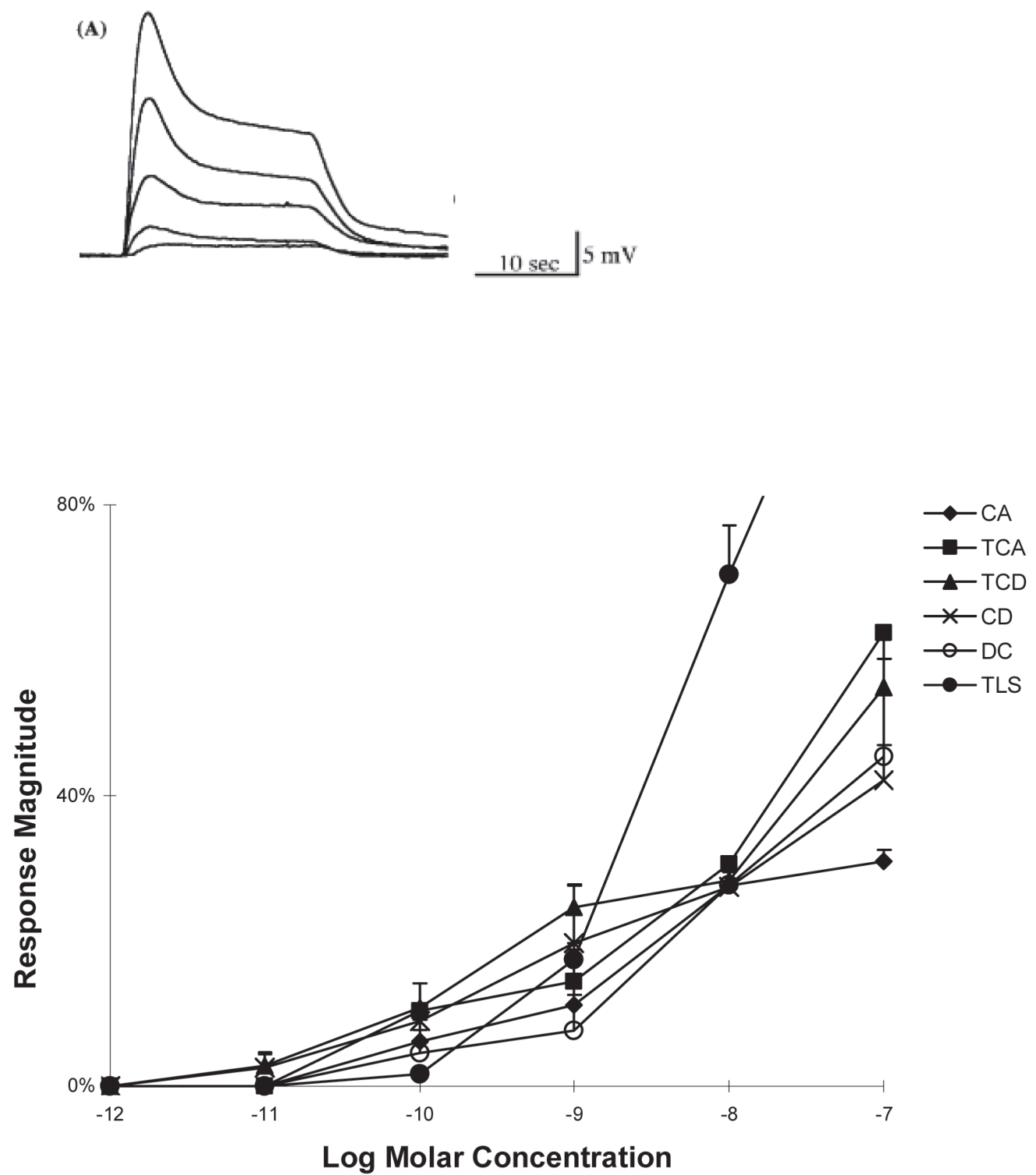

Figure 1: (A) Example tracing of EOG. Superimposed EOG responses to $10^{-9}$ and $10^{-5} \mathrm{M} \mathrm{L}$ cysteine, showing development of tonic and phasic components (scale bar: $10 \mathrm{~s}$ ). (B) Tonic/phasic responses ratios for six representative bile acids at various concentrations. $\mathrm{CA}=$ cholic acid, TCA=taurocholic acid, TCD=taurochenodeoxycholic, $\mathrm{CD}=$ chenodeoxycholic, $\mathrm{DC}=\mathrm{acid}$ deoxycholic acid, TLS=taurolithocholic acid 3-sulphate. Responses are expressed as percentages of the response to the standard $10^{-5} \mathrm{M}$ Serine (mean $\pm \mathrm{SEM}$ ). The sample size is 5 . 


\section{Concentration-response relationships}

Concentration-response (C-R) relationships of the six most potent bile acids tested were grouped according to the molecular structures (Fig. 2). Three groups were identified, and all compounds within each group had similar C$\mathrm{R}$ relationships. The free bile acids deoxycholic (DC), chenodeoxycholic (CD), and cholic acid (CA) had similar, nearly parallel C-R curves with detection thresholds of $10^{-10} \mathrm{M}, 10^{-11} \mathrm{M}$ and $10^{-10} \mathrm{M}$, respectively (Fig. 2a). The bile acids conjugated with taurine, taurochenodeoxycholic acid (TCD), and taurocholic acid (TCA) had similar C-R curves, with relatively lower detection thresholds of $10^{-11} \mathrm{M}$ and $10^{-10} \mathrm{M}$, respectively (Fig. 2b). Lastly, taurolithocholic acid 3-sulphate (TLS), the only sulfated compound tested, had a C-R curve that was different from all other bile acids (Fig. 2c); it had a detection threshold of $10^{-11} \mathrm{M}$, with a nearly exponential increase between $10^{-10}$ and $10^{-8} \mathrm{M}$.

\section{Cross-adaptation}

When used as adapting stimuli (selfadaptation), all six bile acids eliminated EOG responsiveness to themselves. The results suggested that the interaction of bile acids with receptors is in a reversible and truly competitive manner and may be mediated by specific receptors (Fig. 3).

Adaptation to free bile acid DC inhibited responses to CA and itself (Fig. 3b), but adaptation to $\mathrm{CD}$ did not inhibit TCA responses completely (Fig. 3a), suggesting that separate receptors may exist for DC and $\mathrm{CD}$ and that $\mathrm{DC}$ and $\mathrm{CA}$ share the same receptors. None of the bile acids significantly inhibited TCD responses (other than itself), suggesting separate receptors for TCD. Although TLS responses were suppressed by DC, they were unaffected by others (Fig. 3c). ARG responses were unaffected by all bile acids.

\section{DISCUSSION}

The present study demonstrated that bile acids are a group of potent olfactory stimulants for rainbow trout. This observation is consistent with EOG data in lake char (Zhang et al., 2001), zebrafish (Michel and Lubomudrov, 1995), sea lamprey (Li et al., 1995) and olfactory bulb recording in grayling and Arctic char (Doving et al., 1980).

Our cross-adaptation results indicated a competitive interaction of bile acids with their receptors which is fully reversible at the concentrations range tested. The results suggest that olfactory responses to bile acids may be mediated by specific receptors.

Cross-adaptation is useful in investigating the involvement of single or multiple receptors mediating an observed electrophysiological response. However, the concentration and potency of adapting compounds affect the degree of inhibition to test stimuli. Different experimental strategies and the complicated biological system in in vivo studies sometimes make it difficult to interpret cross-adaptation results (Mair, 1982; Zack-Strausfeld and Kaissling, 1986). For instance, it has been reported that two chemicals with similar concentrations or equipotencies may have different inhibitory power (Cain and Engen, 1969; Caprio and Byrd, 1984). In some cases, more potent compounds have higher adaptation effects on test stimuli (Sugimoto and Sato, 1981; Ohno et al., 1984). Inversely, Baylin and Moulton (1979) concluded that there is no correlation between a compound's potency and crossadaptation power for olfactory responses. Moreover, Zhang et al. (2001) found that strong stimulants are, in general, better adapters for compounds of the same group. Following this hypothesis, in the present study, TCD should inhibit TCA because both are from the same group of amidated bile acids, however this was not evident in the data presented.

In general, the results of previous competing experiments have been interpreted as follows. If the response to an odorant is unaffected by a competitor odorant, it is assumed that the test and competitor odorants interact with distinct transduction pathways including different odorant receptors. Complete and reciprocal cross-adaptation between two odorants is 

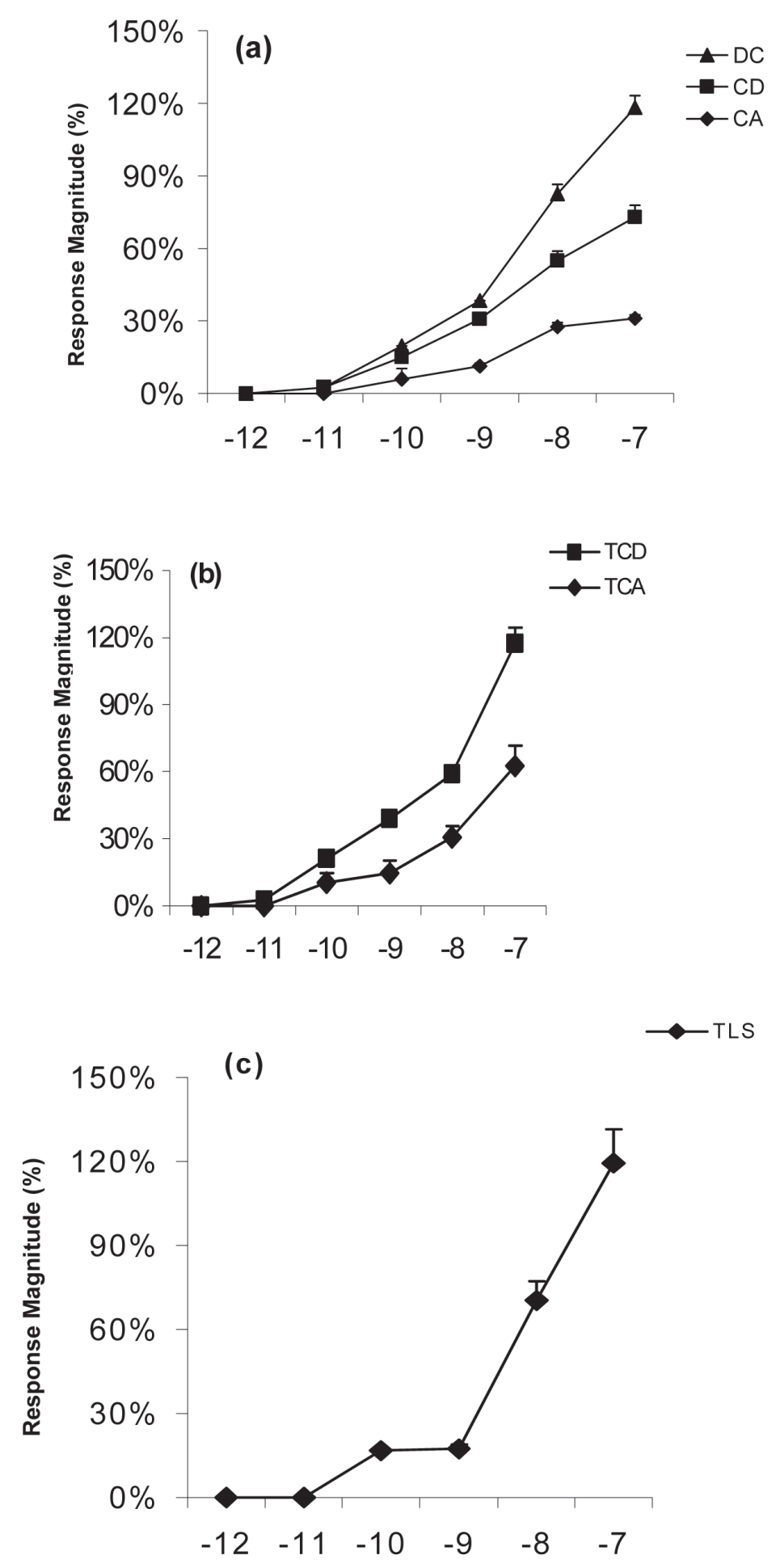

Figure 2: Concentration-response relationships of phasic responses for bile acids. Response magnitudes are normalized as percentages of response to $10^{-5} \mathrm{M}$ L-serine. (a) free bile acids; (b) amidated bile acids; and (c) sulphated bile acids. Responses are expressed as percentages of the response to the standard $10^{-5} \mathrm{M}$ Serine. Each point represents mean \pm S.D. The sample size is 5 . 

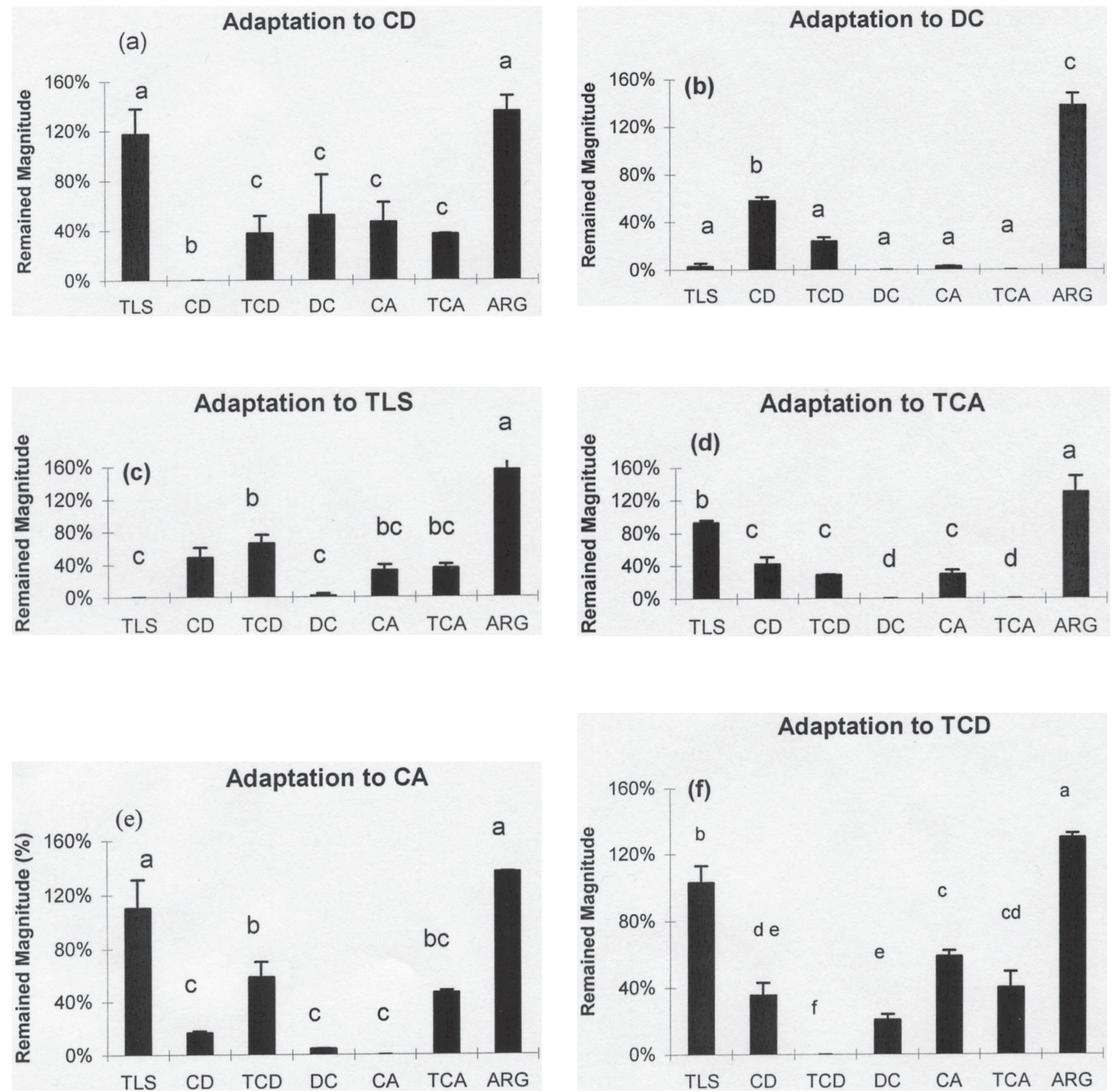

Figure 3: Remained response magnitudes at $10^{-7} \mathrm{M}$ during adaptation to (a) $10^{-7} \mathrm{M}$ chenodeoxycholic acid (CD); (b) $10^{-7} \mathrm{M}$ deoxycholic acid (DC); (c) $10^{-7} \mathrm{M}$ taurolithocholic acid 3sulphate (TLS); (d) $10^{-7} \mathrm{M}$ taurocholic acid (TCA) and (e) $10^{-7} \mathrm{M}$ taurochenodeoxycholic (TCD). Sample size is 5 in a, b, c, e, $\mathrm{f}$ and 4 in d. Responses are expressed as percentages of the response to the standard $10^{-5} \mathrm{M}$ Serine (mean \pm SEM). Different letters above bars indicate a statistically significant difference when compared to the test of adapting stimulus.

interpreted as evidence that the two odorants compete for a common transduction pathway probably including a shared odor receptor.

Partial cross-adaptation, whether reciprocal or not, may be the result of competition for a shared odorant receptor or due to interactions at any step in a shared transduction cascade. Odors that partially, but never completely, cross-adapt each other are common (e.g., CA and DC in this study) and are assumed to reflect interaction at some level of the receptive process.

Similarities and differences in the patterns of cross-adaptation of the test odorants in competitor odorant backgrounds provide information about the probable ligand binding sites of the odorant receptors (Caprio and Byrd, 1984; Ohno et al., 1984). 
Presumably, structurally similar odorants have shared patterns of adaptation because their common structural features activate odorant receptors recognizing those features.

Odorants interacting with a common molecular receptor might be expected to bind to that receptor with different affinities. In some competition studies, the concentrations of the test odorants are adjusted until approximately equal response magnitudes are obtained in an attempt to correct for differences in binding affinity (Caprio and Byrd, 1984). Cross-adaptation between odorants presumed to interact with common receptor might not be expected to be reciprocal if their concentrations had not been so adjusted.

Our data showed that even when the adapting compound and the test stimuli were from the same group, there was no inhibition. This could mean that the inhibition (or lack of inhibition) of the compounds is group independent. For example, the free bile acids DC and CA were not even slightly inhibited when the adapting compound was another free bile acid (CD), whereas DC was totally inhibited when the adapting compound was TLS, a sulphated bile acid. This suggests that the adapting compound acts as a partial agonist that competes for receptors of other groups of bile acids. If there are multiple bile acid receptors, then it is expected that a bile acid would interact with more than one type of receptor because molecular structures among bile acids groups are similar.

Analyses of structure-activity relationship suggest that certain configurations are important for individual receptor recognition. For example, the position and orientation of hydroxyls presented on the ring structure affect the stimulus effectiveness. It appears that a-axial formed by hydroxyls of 3 a- and 7 a- is important in the stimulatory potency of the compound. These features suggest that bile acids interact with their receptor in pointedly positions rather than in collective effects.

Among bile acids, the most potent are CA, TCA, TCD, CD, DC, and TLS. At least three types of bile acid receptors are responsible for olfactory responses to these most stimulatory bile acids. However, a bile acid may react with one or more bile acid receptors.

$\mathrm{Li}$ and Sorensen (1994) suggest that there are four types of bile acid receptors in the sea lamprey olfactory epithelium. Our results appear similar to those reported in sea lamprey. Thus, it seems that bile acid receptors exist in many fish species, but it is yet to be determined if similar receptors present in a wide range of species have significance in chemical signal detection.

\section{Implication of bile acids as chemical signals}

In salmonids, there is little degradation of bile acids in the intestine. It is thought that the extensive bile acid metabolism occurring in the mammalian intestine is caused by microorganisms (Gustafsson et al., 1968; Huijghebaert and Hofmann, 1986). In fish, especially in salmonides, the occurrence of fewer intestinal microorganisms probably accounts for the lack of metabolic transformation of bile acids (Trust and Sparrow, 1974; Trust, 1975).

Fish utilize bioactive byproducts as chemical signals for communication and the olfactory system plays a predominant role in perception of chemical signals (Hara, 1982; Hara, 1992). Feces and urine represent two sources for these bioactive byproducts in water. Although the ecological significance of bile acids in feces, urine and water remains to be established, spawning lake char are attracted to reefs treated with feces of juvenile lake char. Females approach and males contact the treated reefs, and successful spawning was observed at these sites. This suggests that chemical cues emanating from feces of juvenile lake char may play a role in the reproductive behavior of spawning adults. This is consistent with the evidence that chemical signals are often a mixture of related chemical (Sorensen et al., 1998). Presumably organisms have evolved to discern pheromonal mixtures because they contain more information and represent a more portent signal (Sorensen et al., 2003). Thus, a small portion of certain bile acid 
components may be important in forming chemical cues. Experiments are presently underway to confirm the precise behavioral significance of these components.

\section{ACKNOWLEDGEMENTS}

This study was funded by a Fundação para o Amparo da Pesquisa do Estado de São Paulo, FAPESP post-doctoral fellowship to P.C.G (process number: 02/01333-9), Brazil and the Canada Department of Fisheries and Oceans. All experimental procedures employed in this study complied with the Canadian Council for Animal Care guidelines.

\section{REFERENCES}

BAYLIN F, MOULTON DG (1979) Adaptation and crossadaptation to odor stimulation of olfactory receptors in the tiger salamander. J Gen Physiol 74: 37-55

CAIN WS, ENGEN T (1969) Olfactory adaptation and the scaling of odor intensity. In: PFAFFMANN C (ed) Olfaction and Taste III. New York: Rockefeller University Press. pp: 127-141

CAPRIO J, BYRD RP (1984) Electrophysiological evidence for acidic, basic, and neutral amino acid olfactory receptor sites in the catfish. J Gen. Physiol 84: 403-422

DENTON JE, YOUSEF MK (1974) Bile acid composition of rainbow rrout, Salmo gairdneri. Lipids 9: 945-951

DOVING KB, SELSET R, THOMMESEN G (1980) Olfactory sensitivity to bile acids in salmonid fishes. Acta Physiol Scand 108: 123-131

GOTO T, UI T, UNE T, KURAMOTO T, KIRIRA K, HOSHITA $T$ (1996) Bile salt composition and distribution of the D-cystenolic acid conjugated bile salts in fish. Fish Sci 62: 606-609

GUSTAFSSON BE, MIDTVEDT T, NORMAN S (1968) Metabolism of cholic acid in germ free animals after establishment in the intestinal tract of deconjugating and 7a-dehydroxylating bacteria. Acta Pathol Microbiol Scand 72: 433-443

HARA TJ, LAW YMC, VAN DER VEEN E (1973) A stimulatory apparatus for studying the olfactory activity in fishes. J Fish Res Bd Can 30: 282-285

HARA TJ (1982) Chemoreception in Fishes. Amsterdam: Elsevier

HARA TJ, MCDONALD S, EVANS RE, MARUI T, ARAI S. (1984) Morpholine, bile acids, and skin mucus as possible chemical cues in salmonid homing: Electrophysiological re-evaluation. In: MCCLEAVE JD, ARNOLD JP, DODSON JJ, NEILL WH (eds) Mechanisms of migration in fishes. New York: Plenum. pp: 363-378

HARA TJ (1992) Fish Chemoreception. London: Chapman and Hall

HARA TJ (1994a) The diversity of chemical stimulation in fish olfaction and gustation. Rev Fish Biol Fish 4: 1-35

HARA TJ (1994b) Olfaction and gustation in fish: An overview. Acta Physiol Scand 152: 207-217
HARA TJ, ZHANG C (1996) Olfactory responses to putative pheromones and their neural pathways in salmonids. In: CANARIO AVM, POWER DM (comp) Proceedings of the workshop "Fish Pheromones: Origins and Mechanisms of Action," University of Algarve, Faro, 22-40 May 1995, Faro, Portugal, 82-97

HASLEWOOD, GAD (1967) Bile salts. London: Methuen

HUIJGHEBAERT SM, HOFMANN AF (1986) Pancreatic carboxypeptidase hydrolyses of bile acid-amino acid conjugates: Selective resistance of glycine and taurine amidates. Gasteroenterology 90: 306-315

LI W, SORENSEN PW (1994) High specificity of the sea lamprey olfactory system to four classes of bile acids. Chem Senses 19: 506

LI W, SORENSEN PW, GALLAHER DD (1995) The olfactory system of migratory adult sea lamprey (Petromyzon marinus) is specifically and acutely sensitive to unique bile acids released by conspecific larvae. J Gen. Physiol 105: 569-587

MAIR RG (1982) Adaptation of rat olfactory bulb neurons. J Physiol 26: 361-369

MICHEL WC, LUBOMUDROV LM (1995) Specificity and sensitivity of the olfactory organ of the zebrafish, Danio rerio. J Comp Physiol A 177: 191-199

QUINN TP, HARA TJ (1986) Sibling recognition and olfactory sensitive in juvenile coho salmon (Oncorhynchus kisutch). Can J Zool 64: 921-925

OHNO T, YOSHII K, KURIHARA K (1984) Multiple receptor types for amino acids in the carp olfactory cells revealed by quantitative cross-adaptation method. Brain Res 310: 13-21

OTTOSON D. (1971) The electro-olfactogram. In: BEIDLER LM (ed). Handbook of Sensory Physiology. Berlin: Springer, vol. 4. pp: 95-13

SOLA C, TOSI L (1993) Bile salts and taurine as chemical stimuli for glass eel, Anguilla anguilla: a behavioral study. Environ Biol Fishes 37: 197-204

SORENSEN PW, HARA TJ, STACEY NE (1987) Extreme olfactory sensitivity of mature and gonadally-regressed goldfish to a potent steroidal pheromone, 17a, 20bdihydroxy-4-pregnen-3-one. J Comp Physiol 160: 305313

SORENSEN PW, CHRISTENSES TA, STACEY NE (1998) Discrimination of pheromonal cues in fish: emerging parallels with insect. Curr Opin Neurobiol 13: 689-695

SORENSEN PW, VRIEZE LA, FINE JM. (2003) A multicomponent migratory pheromone in the sea lamprey. Fish Physiol Biochem 28: 253-257

STABELL OB (1992) Olfactory control of homing behavior in salmonids. In: HARA TJ (ed) Fish Chemoreception. London: Chapman and Hall. pp: 249270

SUGIMOTO K, SATO T (1981) The adaptation of the frog tongue to bitter solutions: The effect on gustatory neural responses to acid, sugar and bitter stimuli. Comp Biochem Physiol 73A: 361-372

SVEINSSON T, HARA TJ (1990) Multiple olfactory receptors for amino acids in Arctic char (Salvelinus alpinus) evidenced by cross adaptation experiments. Comp Biochem Physiol 97A: 289-293

TRUST TJ, SPARROW RAH (1974) The bacterial flora in the alimentary tract of freshwater salmonid fishes. Can J Microbiol 20: 1219-1228

TRUST TJ (1975) Facultative anaerobic bacteria in the digestive tract of chum salmon (Oncohynchus keta) maintained in fresh water under defined culture conditions. Appl Microbiol 29: 663-668

UNE M, GOTO T, KIRIRA K, KURAMOTO T, HAGIWARA K, NAKAGIMA T, HOSHITA T (1991) 
Isolation and identification of bile salts conjugated with cysteinolic from bile of the red seabream, Pagrosomus major. J Lipid Res 32: 1619-1623

ZACK-STRAUSFELD C， KAISSLING KE (1986) Localized adaptation process in olfactory sensilla of Saturnid moths. Chem Senses 11: 449-512

ZHANG C, HARA TJ (1991) Olfactory and gustatory responses to bile salts in salmonids. In: Chemical Signals in Vertebrates VI. Philadelphia, Pennsylvania, USA, June 16-22

ZHANG C, SCOTT BB, HARA TJ (2001) Biochemical and physiological evidence that bile acids produced and released by lake char (Salvelinus namaycush) function as chemical signals. J Comp Physiol B 171: 161-171 